\title{
Mechanism for a Decaying Cosmological Constant
}

\author{
Y. Bisabr 丹, H. Salehi [ \\ Department of Physics, Shahid Beheshti University, Evin, Tehran 19839, Iran.
}

\begin{abstract}
A mechanism is introduced to reduce a large cosmological constant to a sufficiently small value consistent with the observational upper limit. The basic ingradient in this mechanism is a distinction which has been made between the two unit systems used on cosmology and particle physics. We have used a conformal invariant gravitational model to define a particular conformal frame in terms of the large scale properties of the universe. It is then argued that the contributions of mass scales in particle physics to the vacuum energy density should be considered in a different conformal frame. In this manner a cancellation mechanism is presented in which the conformal factor plays a key role to relax the large effective cosmological constant.
\end{abstract}

Cosmological observations imply that there exists an extremely small upper limit on the vacuum energy density in the present state of our universe. This stands in sharp contradiction with theoretical predictions [1]. In fact, any mass scale in particle physics contributes to the vacuum energy density much larger than this upper bound [2]. It therefore seems that a, yet unknown, cancellation mechanism must take place to reduce these huge contributions to a sufficiently small value. One may expect that such a mechanism has two characterictics. Firstly, it should not be sensitive to a particular type of contribution and should work equally well for every mass scale introduced by particle physics. Secondly, it should work whenever these contributions are considered at cosmological level since the discrepancy manifests when one compares them with relevant cosmological observations. This latter strongly suggests that construction of a mechanism for relaxing these contributions should somehow take into account the distinction between the two unit systems defined by cosmology and particle physics. One

\footnotetext{
*e-mail: y-bisabr@cc.sbu.ac.ir.

†e-mail: h-salehi@cc.sbu.ac.ir.
} 
should note that the small upper limit is obtained in a unit system which is defined in terms of large scale cosmological parameters. On the other hand, the theoretical predictions are based on a natural unit system which is suggested by quantum physics. With this fact in mind, the point we wish to make here is that the large descrepancy, which is usually known as the cosmological constant problem, arises when one prejudices that the two unit systems should be indistinguishable up to a constant conversion factor in all spacetime points. It means that they should transform to each other by a global unit transformation. Such a global transformation clearly carries no dynamical implications and the use of a particular unit system is actually a matter of convenience. It means that one may arbitrarily use the unit system suggested by quantum physics to describe the evolution of the universe or the cosmological unit system to describe dynamical properties of an elementary particle.

In this Letter we introduce a theoretical scheme in which an explicit recognition is given to the distinguished characteristics of these two unit systems. In such a theoretical shceme one should no longer accept the triviality one usually asigns to a unit tranformation. In this respect, we shall consider local unit transformations, conformal transformations [3], which relates different standard of units, conformal frames, via general spacetime dependent conversion (conformal) factors. In this language the observational and the theoretical predictions on the vacuum energy density are actually carried out in two different conformal frames. We emphasize that local unit transformations give a dynamical meaning to changes of unit systems and can be consequently taken as a basis for constructing a dynamical mechanism which works due to cosmic expansion. Along this line of investigation, we shall show that the conformal factor which relates the two unit systems plays the role of a dynamical field which can eventually reduce the effective cosmological constant to a small value consistent with observations. To begin with, let us cosider a gravitational model described by the action

$$
S=\frac{1}{2} \int d^{4} x \sqrt{-g}\left(g^{\alpha \beta} \nabla_{\alpha} \phi \nabla_{\beta} \phi+\frac{1}{6} R \phi^{2}\right)+S_{m}\left(g_{\alpha \beta}\right)
$$

in which $\nabla_{\alpha}$ denotes a covariant differentiation, $\phi$ is a scalar field, $R$ is the Ricci scalar and $S_{m}\left(g_{\alpha \beta}\right)$ is the action of some matter fields. The gravitational part of this action remains invariant under conformal transformations

$$
\begin{gathered}
\bar{g}_{\alpha \beta}=e^{2 \sigma} g_{\alpha \beta} \\
\bar{\phi}=e^{-\sigma} \phi
\end{gathered}
$$

where $\sigma$ is a smooth dimensionless spacetime function. Variation with respect to $g^{\alpha \beta}$ and $\phi$ yields, respectively,

$$
\begin{gathered}
G_{\alpha \beta}=6 \phi^{-2}\left(T_{\alpha \beta}+\tau_{\alpha \beta}\right) \\
\square \phi-\frac{1}{6} R \phi=0
\end{gathered}
$$

where

$$
\tau_{\alpha \beta}=-\left(\nabla_{\alpha} \phi \nabla_{\beta} \phi-\frac{1}{2} g_{\alpha \beta} \nabla_{\gamma} \phi \nabla^{\gamma} \phi\right)+\frac{1}{6}\left(\nabla_{\alpha} \nabla_{\beta}-g_{\alpha \beta} \square\right) \phi^{2}
$$

\footnotetext{
[5].

${ }^{\ddagger}$ Throughout this paper we work in units in which $\hbar=c=1$ and the sign conventions are those of MTW
} 


$$
T_{\alpha \beta}=\frac{-2}{\sqrt{-g}} \frac{\delta}{\delta g^{\alpha \beta}} S_{m}\left(g_{\alpha \beta}\right)
$$

Here $\square \equiv g^{\alpha \beta} \nabla_{\alpha} \nabla_{\beta}$ and $G_{\alpha \beta}$ is the Einstein tensor. If one substitutes the equation (5) into the trace of (4) one obtains $T_{\gamma}^{\gamma}=0$ which means that the stress tensor of matter must be traceless. This is a consequence of the absence of a dimensional parameter in the gravitational part of the action (四). One may consider matter systems with nonvanishing trace by introducing a term such as

$$
-\frac{1}{6} \int d^{4} x \sqrt{-g} \mu^{2} \phi^{2}
$$

in which $\mu$ is a parameter with dimension of mass. In this case, one obtains

$$
\begin{gathered}
G_{\alpha \beta}+\mu^{2} g_{\alpha \beta}=6 \phi^{-2}\left(T_{\alpha \beta}+\tau_{\alpha \beta}\right) \\
\square \phi-\frac{1}{6} R \phi+\frac{1}{3} \mu^{2} \phi=0
\end{gathered}
$$

and

$$
T_{\gamma}^{\gamma}=\frac{1}{3} \mu^{2} \phi^{2}
$$

The parameter $\mu$ also allows us to study breakdown of the conformal invariance in this model. In general, in a conformal transformation all dimensional parameters are required to be transformed according to their dimensions so that $\mu$ should obey the transformation rule $\mu \rightarrow e^{-\sigma} \mu$. The conformal invariance can, however, be broken when a particular conformal frame is chosen in which the dimensional parameter takes on a constant configuration. The choice of such a specific conformal frame, or equivalently the constant value attributed to $\mu$, is merely suggested by the physical conditions one wishes to investigate in the problem at hand. In the conformal frame which is introduced in this way the gravitational coupling is then characterized by the preferred configuration of $\phi^{-2}$.

Here we first intend to determine a conformal frame with use of the large scale properties of the observed universe. In this case, the most suggestive choice for $\mu$ is $\mu \sim H_{0}$ with $H_{0}^{-1}$ being the present Hubble radius of the universe. In the corresponding conformal frame, which is referred from now on as the cosmological frame, we try then to find the preferred constant configuration of $\phi^{-2}$ in terms of large scale distribution of matter contained in the universe. To do this, we take $T_{\alpha \beta}$ to be a pressureless perfect fluid with energy density $\rho$ and write the gravitational equations (9) for a spatially flat Friedman-Robertson-Walker metric

$$
d s^{2}=-d t^{2}+a(t)\left(d x^{2}+d y^{2}+d z^{2}\right)
$$

where $a(t)$ is the scale factor. In the present epoch of evolution of the universe this leads to

$$
H_{0}^{2} \sim \rho_{0} \phi^{-2}
$$

in which $\rho_{0}$ is the present energy density of cosmic matter. We also use the observational fact that $\rho_{0} \sim \rho_{c}$ which $\rho_{c}=\frac{3 H_{0}^{2}}{8 \pi G}$ is the critical density. The relation (13) then gives

$$
\phi^{-2} \sim G
$$


Thus in the cosmological frame $\phi^{-2}$ takes a constant configuration which is given by the gravitational constant. By implication the action (1), together with (8), reduces to

$$
S=\frac{1}{16 \pi G} \int d^{4} x \sqrt{-g}\left(R-2 \mu^{2}\right)+S_{m}\left(g_{\alpha \beta}\right)
$$

This corresponds to the usual Einstein-Hilbert action with a cosmological constant induced due to finite size of the universe.

The cosmological constant, however, receives strong contributions from various mass scales introduced by particle physics. To clarify how these contributions enter our gravitational model in the cosmological frame, it should be remarked that the conformal invariance breaking might be considered as a result of emergence of rest masses of elemetary particles in appropriately small energy scales. One could therefore define an alternative conformal frame in which these masses take some constant values. Such a conformal frame would evidently have properties entirely different from those that used to define the cosmological frame. In particular, it should be defined in terms of local characteristics of a typical elementary particle and should neglect the large scale properties of the universe. It follows that when one compares contributions of the rest masses to vacuum energy density with relevant cosmological observations one should note the fact that they belong to two different conformal frames. This means that the rest masses should have a variable contribution to vacuum energy density in the cosmological frame. We would like to analze this assertion by writing the action (15) in the form

$$
S=\frac{1}{16 \pi G} \int d^{4} x \sqrt{-g}\left\{R-2\left(\mu^{2}+\bar{\Lambda}\right)\right\}+S_{m}\left(g_{\alpha \beta}\right)
$$

where $\bar{\Lambda}=e^{-2 \sigma} \Lambda$ and $\Lambda$ being a typical mass scale in particle physics. Since the conformal factor appears here as a new dynamical degree of freedom we let the above action involve a kinetic term for $\sigma$ so that

$$
S=\frac{1}{16 \pi G} \int d^{4} x \sqrt{-g}\left\{R-2\left(\mu^{2}+\Lambda e^{-2 \sigma}\right)-\epsilon g^{\alpha \beta} \nabla_{\alpha} \sigma \nabla_{\beta} \sigma\right\}+S_{m}\left(g_{\alpha \beta}\right)
$$

where $\epsilon$ is a dimensionless constant parameter of order of unity. In this way for $\epsilon>0, \sigma$ acts as a normal dynamical field with positive energy density which allows us to investigate the evolution of $\bar{\Lambda}$ as the universe evolves. We now follow the consequences of the action (17) by writing the field equations

$$
\begin{gathered}
G_{\alpha \beta}+\left(\mu^{2}+\Lambda e^{-2 \sigma}\right) g_{\alpha \beta}-\epsilon\left(\nabla_{\alpha} \sigma \nabla_{\beta} \sigma-\frac{1}{2} g_{\alpha \beta} \nabla^{\gamma} \sigma \nabla_{\gamma} \sigma\right)=8 \pi G T_{\alpha \beta} \\
\square \sigma+\frac{2}{\epsilon} \Lambda e^{-2 \sigma}=0
\end{gathered}
$$

In these equations the exponential coefficient for $\Lambda$ emphasizes the distinction between the two unit systems mentioned above. In an expanding universe this distinction is expected to increase since cosmological scales enlarge as the universe expands and the conformal factor $e^{2 \sigma}$ must grow with time. This authomatically provides us with a dynamical reduction of $\bar{\Lambda}$ in the cosmological frame. That this intuitive picture is actually consistent with the field equations 
is illustrated in the following:

Applying (18) and (19) to the metric (12), yields

$$
\begin{gathered}
3 H^{2}-\Lambda_{e f f}=8 \pi G \rho \\
\dot{\rho}+3 H \rho=0 \\
\ddot{\sigma}+3 H \dot{\sigma}-\frac{2}{\epsilon} \Lambda e^{-2 \sigma}=0
\end{gathered}
$$

with

$$
\Lambda_{e f f}=\mu^{2}+\Lambda e^{-2 \sigma}+\frac{1}{2} \epsilon \dot{\sigma}^{2}
$$

where $H=\frac{\dot{a}}{a}$ and the overdot indicates differentiation with respect to $t$. Due to homogeneity and isotropy, the field $\sigma$ is taken to be only a fuction of time.

The equation (21) immediately gives

$$
\rho a^{3}=\text { const }
$$

Assuming that the universe follows a power law expansion, namely that $H \sim t^{-1}$, we use the ansatz

$$
e^{\sigma}=\sigma_{0} t
$$

which satisfies the equation (22) with $\sigma_{0} \sim \sqrt{\Lambda}$. From (23) one then obtains $\Lambda_{e f f} \sim t^{-2}$ which gives the observational upper limit. With this result, the equation (20) suggests that $\rho \sim t^{-2}$ which is consistent with evolution of matter density in the standard cosmological model.

In summary, it is argued that different contributions to the energy content of vacuum may come from different conformal frames. In particular, we have shown that the rest masses of elementary particles should be regarded as contributions coming from a conformal frame with properties different from those that suggested by cosmological observations. This line of reasoning then leads us to introduce a mechanism in which the conformal factor appears as a dynamical field and makes a large cosmological constant damp during evolution of the universe. It should be remarked that the nontrivial behaviour of the conformal factor in such a mechanism may have notable outcomes at early universe. For instance, it is worthwhile to study the effect of such a dynamical field on the inflationary scenarios or on the possible avoidance of the cosmological singularity. We intend to investigate these issues elsewhere.

\section{References}

[1] S. Weinberg, Rev. Mod. Phys. 61, 1 (1989)

[2] H. Salehi, Int. J. Theor. Phys. 36, 2035 (1997)

[3] J. D. Bekenstein and A. Meisels, Phys. Rev. D 22, 1313 (1980)

[4] H. Salehi, Y. Bisabr and H. Ghafarnejad, J. Math. Phys. 41, 4582 (2000)

H. Salehi and Y. Bisabr, Int. J. Theor. Phys. 39, 1241 (2000)

S. Deser, Ann. Phys. 59, 248 (1970)

H. Salehi, H. Sepangi and F. Darabi, Founds. Phys. Lett. 13, 297 (2000) 
[5] C. W. Misner, K. S. Thorne and J. A. Wheeler, Gravitation (Freeman, San Francisco, 1973) 